was relatively uncommon and one third required a stoma at surgery. Variation between Trusts in coding quality is inevitable but the data suggest 1 in 5 institutions may lack provision for SEMS.

Competing interests J Geraghty: Grant/Research Support from: Cook Medical, S Sarkar: None declared, M Shawihdi: None declared, E Thompson: None declared, M Pearson: None declared, K Bodger: None declared.

\section{PTU-214 AN IN-VITRO STUDY TO ASSESS, AND IMPROVE, THE ACCURACY OF COLONIC POLYP SIZING AMONG NURSE ENDOSCOPISTS, TRAINEES AND CONSULTANT GASTROENTEROLOGISTS}

doi:10.1136/gutjnl-2012-302514c.214

J Geraghty, ${ }^{*}$ Z Raisi-Estabragh, M Gillon, P O’Toole, S Sarkar. Gastroenterology, Royal Liverpool University Hospital, Liverpool, UK

Introduction Knowing if polyps are larger than $10 \mathrm{~mm}$ is critical when determining colonoscopic surveillance strategies. Judging polyp size from the endoscopic view alone becomes important if polyps are not retrieved intact. Strategies based on deliberately discarding small polyps rely on accurate discrimination of polyp size but little is known about endoscopists ability to make this judgement. Our aim is to assess the accuracy of polyp size estimation using a novel in vitro model, comparing different professional groups and use of accessories to improve estimates.

Methods Nine endoscopists ( 3 consultants, 3 trainees and 3 nurse endoscopists) judged the size of 15 "polyps" made from modelling clay (size range $6-36 \mathrm{~mm}$ ) placed inside a colonoscopy training model (Koken Co Ltd, Tokyo). Polyps of different sizes were presented in random order. Size estimates were made using endoscopic visual assessment alone or by comparing the polyp to biopsy forceps or a $10 \mathrm{~mm}$ snare. A degree of confidence for each guess was recorded.

Results Consultants and trainees were significantly better than nurse endoscopists at judging whether the model polyps were larger or smaller than $10 \mathrm{~mm}(91.8 \%$ vs $79.2 \% \mathrm{p}<0.05)$. Overall, visual assessment alone had an accuracy of $78.8 \%$. Inaccuracy was largely due to underestimation of size. Use of accessories improved discrimination around the $10 \mathrm{~mm}$ threshold $(\mathrm{p}<0.05)$. The snare produced slightly better accuracy (87.9\%) than forceps $(83.8 \%)$ (NS). All professional groups expressed similar degrees of confidence in their estimates.

Conclusion In this model, medical endoscopists were better than nurse endoscopists in assessing the size of polyps. This may be because nurses in our study do not routinely perform polypectomy whereas doctors have all had the opportunity to learn from comparing the size of resected polyps with their original endoscopic assessment. Use of biopsy forceps or a snare improved size estimation and these may be helpful tools when teaching this important aspect of polyp assessment in vivo.

Competing interests J Geraghty Grant/Research Support from: Cook Medical, Z Raisi-Estabragh: None declared, M Gillon: None declared, P O'Toole: None declared, S Sarkar: None declared.

\section{PTU-215 ASSESSING COMFORT SCORES FOR CO2 INSUFFLATION AS COMPARED TO AIR IN FLEXIBLE SIGMOIDOSCOPY-A PROSPECTIVE AUDIT}

doi:10.1136/gutjnl-2012-302514c.215

J Tharakan, ${ }^{*}$ D Beesley, A Kelling, R Radzioch, I Tiwari. Endoscopy unit, Braintree Community hospital, Essex, UK

Introduction Patients experience of discomfort with Air insufflation during flexible sigmoidoscopy (FS) limits compliance and thus success of the procedure. There has been only one study ${ }^{1}$ which has shown that CO2 insufflation reduces discomfort as compared to Air in FS. Recently, we have been using CO2 insufflation for routine FS. We therefore conducted a prospective audit comparing the two modalities and to assess whether the use of $\mathrm{CO} 2$ during FS reduces discomfort both during and after the procedure using a standardised scoring system.

Methods 200 consecutive patients undergoing FS, commonly for rectal bleeding, altered bowel habit and abdominal pain were selected to either Air or CO2 insufflation. There were 100 patients (42 males) in the CO2 group and 100 patients (51 males) in the Air group. The ages ranged from 19 to 92 years in both the groups. Any history of previous abdominal surgery was also noted. Patients were asked to grade discomfort during the procedure, post procedure in the recovery room and on discharge. We used the standardised comfort score of Wong and Baker $(0==$ no discomfort and $10=$ extreme discomfort). Abdominal bloating was also assessed verbally after the procedure. Statistical analysis was done using Prism software.

Results The mean comfort scores for $\mathrm{CO} 2$ compared to Air during the procedure was 1.02 vs 1.93 ( $p=0.0006)$, postprocedure 0.54 vs $1.12(p=0.002)$ and on discharge 0.32 vs $0.8(p=0.0008)$ respectively. Abdominal bloating appeared to be less with $\mathrm{CO} 2$ as compared to Air on verbal questioning. No differences in comfort scores were observed with a history of previous abdominal surgery.

Conclusion This study has shown that $\mathrm{CO} 2$ insufflation reduces discomfort as compared to Air during FS, both during and after the procedure. Abdominal bloating was also significantly reduced. The use of CO2 will contribute to better public acceptance for FS, in particular for FS screening in colorectal cancer.

Competing interests None declared.

\section{REFERENCE}

1. Bretthauer M, Hoff G, Thiis-Evensen, et al. CO2 insufflation reduces discomfort due to flexible sigmoidoscopy. Scan J Gastroenterol 2002;37:1103-7.

\section{PTU-216 A SURVEY OF PATIENTS ATTITUDES TO COLONOSCOPY DEMONSTRATES HIGH VALUE FOR ENDOSCOPIST INTERACTION BUT NOT THE SINGLE SEX ENVIRONMENT}

doi:10.1136/gutjnl-2012-302514c.216

J McEntire, ${ }^{*}$ J Sahota, T Hyde, T Trebble. Portsmouth Hospitals NHS, Portsmouth, UK

Introduction Understanding patient attitudes to their medical experience is essential for optimising care and use of resources. This includes their interaction with their health practitioner and their healthcare environment. This study was undertaken to determine patient's preferences and expectations for outpatient colonoscopy, a common gastrointestinal procedure for which there is limited such data from the UK.

Methods Unselected patients attending for elective colonoscopy at a large District General Hospital on randomly selected days in October and November 2011 were invited to participate. Patients independently completed a composite, validated dedicated endoscopy questionnaire, with Likert scale anxiety-related and single sex environment questions and a 15-point preference (ranking) scale of aspects of endoscopy care that were considered most important (1) to least important (15) as contributing to a satisfactory experience. Qualitative and pilot studies were performed initially to confirm validity and reliability in the local population.

Results 217 out of 225 patients agreed to participate (96.4\%); male (49\%) and female (51\%), with mean age of 58 years (range 16-87 years). Mild to moderate anxiety was recorded in over $70 \%$ of 